\title{
Simian Immunodeficiency Virus Utilizes Human and Sooty Mangabey but Not Rhesus Macaque STRL33 for Efficient Entry
}

\author{
STEFAN PÖHLMANN, ${ }^{1}$ BENHUR LEE, ${ }^{2}$ SILKE MEISTER, ${ }^{1}$ MANDY KRUMBIEGEL, ${ }^{1}$ \\ GEORGE LESLIE, ${ }^{2}$ ROBERT W. DOMS, ${ }^{2}$ AND FRANK KIRCHHOFF ${ }^{1 *}$ \\ Institute for Clinical and Molecular Virology, University of Erlangen-Nürnberg, 91054 Erlangen, Germany, ${ }^{1}$ \\ and Department of Pathology and Laboratory Medicine, University of Pennsylvania, \\ Philadelphia, Pennsylvania $19104^{2}$
}

Received 5 January 2000/Accepted 7 March 2000

\begin{abstract}
It has been established that many simian immunodeficiency virus (SIV) isolates utilize the orphan receptors GPR15 and STRL33 about as efficiently as the chemokine receptor CCR5 for entry into target cells. Most studies were performed, however, with coreceptors of human origin. We found that SIV from captive rhesus macaques (SIVmac) can utilize both human and simian CCR5 and GPR15 with comparable efficiencies. Strikingly, however, only human STRL33 (huSTRL33), not rhesus macaque STRL33 (rhSTRL33), functioned efficiently as an entry cofactor for a variety of isolates of SIVmac and SIV from sooty mangabeys. A single amino acid substitution of S30R in huSTRL33 impaired coreceptor activity, and the reverse change in rhSTRL33 greatly increased coreceptor activity. In comparison, species-specific sequence variations in $\mathbf{N}$-terminal tyrosines in STRL33 had only moderate effects on SIV entry. These results show that a serine residue located just outside of the cellular membrane in the $\mathbf{N}$ terminus of STRL33 is critical for SIV coreceptor function. Interestingly, STRL33 derived from sooty mangabeys, a natural host of SIV, also contained a serine at the corresponding position and was used efficiently as an entry cofactor. These results suggest that STRL33 is not a relevant coreceptor in the SIV/macaque model but may play a role in SIV replication and transmission in naturally infected sooty mangabeys.
\end{abstract}

Simian immunodeficiency virus (SIV) from captive rhesus macaques (SIVmac) and human immunodeficiency virus type 2 (HIV-2) show a high degree of genetic homology to SIV in naturally infected sooty mangabeys (SIVsm), suggesting that both originated from SIVsm by cross-species transmission (35, 36). SIV and HIV-2 show many similarities to HIV-1, the etiologic agent of AIDS $(11,49)$. SIVsm causes a persistent but asymptomatic infection in its natural African host (55). HIV-2 is pathogenic in humans, but infected individuals exhibit long clinical latency periods and progress slowly toward AIDS (18, 49). In contrast, SIVmac-infected rhesus macaques frequently develop an AIDS-like disease within the first year after infection $(17,40,45)$. Therefore, infection of macaques with SIVmac represents the most commonly used animal model for the study of AIDS pathogenesis, therapy, and vaccine development $(21,39)$.

Entry of both HIV and SIV into target cells usually involves the binding of the external envelope glycoprotein (gp120) to the cellular CD4 receptor molecule and subsequent interactions with seven transmembrane-spanning G-protein-coupled chemokine receptors (7TM GPCRs), which mediate fusion between the viral and host cell membrane (reviewed in references $8,9,22,51$, and 68). CCR5 and CXCR4 are the major HIV-1 coreceptors $(2,15,19,23,24,34)$. HIV-2 isolates are less restricted to CXCR4 and CCR5 and frequently utilize additional coreceptors $(13,54,57)$. In contrast to both HIV-1 and HIV-2, SIV isolates use CCR5 but usually not CXCR4 for viral entry $(12,13,37,41,48)$, although macaque- and mangabey-derived CXCR4 is fully functional for HIV-1 entry (12). The unique exception is SIV from red-capped mangabeys,

* Corresponding author. Mailing address: Institute for Clinical and Molecular Virology, University of Erlangen-Nürnberg, Schlossgarten 4, 91054 Erlangen, Germany. Phone: 49-9131-852-6483. Fax: 49-913185-1002. E-mail: fkkirchh@viro.med.uni-erlangen.de. which uses CCR2B (14). Interestingly, many red-capped mangabeys lack CCR5 due to a naturally occurring polymorphism.

In addition to CCR5, a variety of chemokine and orphan receptors, such as CCR8, GPR15, STRL33, GPR1, ChemR23, and APJ, have been proposed as alternative coreceptors for SIV $(3,16,20,28,29,31,38,46,47,65,66)$. Of these, GPR15 (also named BOB) and STRL33 (also named Bonzo) may be of particular importance in the SIV/macaque model, because both are used nearly as efficiently as CCR 5 by many SIV isolates $(20,28,31)$. STRL33 was identified as an entry cofactor by two independent approaches: degenerate PCR from a tumor-infiltrating cell line (TIL 9) (46) and a functional-expression screening assay for SIV entry cofactors (20). Efficient utilization of STRL33 by HIV-1 is rare and usually only observed at high expression levels $(59,67,69-71)$. Interestingly, however, in a case of vertical mother-child transmission of HIV-1, viral isolates from the infant as well as from the mother efficiently used STRL33 for entry $(70,71)$. The CCR5-, CXCR4-, and STRL33-tropic maternal isolate infected STRL $33^{+}, \mathrm{CCR}^{-}$peripheral blood lymphocytes in the presence of a CXCR4 antagonist, suggesting that STRL33 can mediate HIV-1 entry into primary cells (66a). HIV-2 isolates obtained from patients diagnosed with AIDS frequently and efficiently utilize a broad range of coreceptors, including both STRL33 and GPR15 (13, 54, 57). Blocking experiments suggest that coreceptors other than CCR5 or CXCR4 can mediate HIV-2 replication in primary cells, and it has been proposed that an expanded coreceptor tropism of HIV-2 might correlate with increased pathogenesis (57).

Both GPR15 and STRL33 are expressed by primary cells and in lymphoid tissues and could contribute to viral spread in vivo $(20,66 \mathrm{a})$. The accumulated in vitro data suggest that GPR15 and STRL33 might play a more important role in the pathogenesis of SIV and HIV-2 than in that of HIV-1. Studies on their in vivo relevance, however, are limited. Apparently, 
utilization of GPR15 in addition to CCR5 does not provide a major advantage for SIV replication in vivo (61).

Most of the studies on SIV entry were performed with 7TM GPCRs of human origin. It has been shown, however, that SIVmac utilizes rhesus CCR5 more efficiently than human CCR5 in the absence of CD4 (30). In the present study we investigated whether SIV coreceptor function of GPR15 and STRL33 is species dependent. We show that SIVmac efficiently utilizes human-derived and rhesus macaque-derived CCR5 and GPR15. Unexpectedly, however, human STRL33 (huSTRL33) was used with much higher efficiency as an entry cofactor than rhesus macaque STRL33 (rhSTRL33). This striking result implies that utilization of STRL33 does not play an important role in SIVmac replication in infected rhesus macaques. In contrast, sooty mangabey STRL33 (smSTRL33) was used efficiently by both SIVmac and SIVsm isolates, suggesting that it may be a relevant coreceptor in naturally infected sooty mangabeys.

\section{MATERIALS AND METHODS}

Coreceptor expression vectors. pBabe-puro vectors expressing human and rhesus macaque CCR5 were provided by Nathaniel Landau and Preston Marx through the AIDS Research and Reference Program, Division of AIDS, National Institute of Allergy and Infectious Diseases. Expression vectors for human GPR15 and STRL33 were kindly provided by Dan Littman (Skirball Institute for Molecular Medicine, New York, N.Y.). Vectors expressing rhesus macaque GPR15 were generated as described previously (60). To generate plasmids expressing rhSTRL33, RNA was isolated from rhesus macaque peripheral blood mononuclear cells (PBMC) or the herpesvirus saimiri-transformed rhesus macaque T-cell line 221 (1). cDNA was prepared using an avian myeloblastosis virus reverse transcriptase-based cDNA synthesis kit (Boehringer Mannheim). Primers used for PCR amplification of STRL33 were p5STRL33-comp (5'-CCGGA TCCGGTGTTCATCAGAACAGACACCATG-3') and p3STRL33-comp (5'CCGAATTCTTTCGAAACCCTGGCAAGGCCT- $3^{\prime}$ ). The indicated BamHI and $E c o$ RI restriction sites (underlined) were used for cloning into the pBABEpuro expression vector. The rhSTRL33 amino acid sequences derived from 221 cells and rhesus macaque PBMC were identical to each other and to GenBank sequence AF124380.

smSTRL33 was amplified by PCR from PBMC-derived genomic DNA from three mangabeys using primers p5outSTRL33 (5'-CCAAATATAATTCCTGG GTTCTGACTC-3') and p3outSTRL33 (5'-GCCATGCCTTGCAAATTCCAG AGCAG- $\left.3^{\prime}\right)$. The resulting 1.1-kb amplification products were cloned into the TA cloning vector pCR2.1 (Invitrogen, Carlsbad, Calif.). DNA fragments were excised by EcoRI digestion and cloned into the EcoRI site of the pBABE-puro expression vector. Clones harboring the insert in the correct orientation were identified by restriction analysis. Sequence analysis revealed that clones derived from all animals contained identical inserts representing intact smSTRL33 open reading frames.

Mutagenesis of STRL33. Most STRL33 mutants were generated by PCR mutagenesis using oligonucleotides which introduced nucleotide changes close to the $5^{\prime}$ end and added an AU-1 tag to the $3^{\prime}$ end of the STRL33 coding sequence. The R31S change in rhSTRL33 and the S30R change in huSTRL33 were introduced by overlap extension PCR. For rhSTRL33, the 5' PCR fragment was generated using p5STRL33-comp and p3rhBZ-RS (5'-TTGCTGAACTGCAG GAAGT-3') and the $3^{\prime}$ PCR fragment was generated using p5rhBZ-RS (5'-TT CCTGCAGTTCAGCAAGGTCTTTCTGCC-3') and p3STRL33AU1 (5'-CCG AATTCCTAATGTATCTGTAGGTGTCTAACTGGAACATGCTGGTGGC C- $\left.3^{\prime}\right)$. Both fragments were gel purified and used as the template in a second PCR with p5STRL33-comp and p3STRL33AU1. The S30R mutation was introduced using the same approach, except that oligonucleotides corresponding to the huSTRL33 sequence were used. All PCR-derived fragments were sequenced to ensure that only the intended changes were present.

Virus stocks. Generation of virus stocks was performed by the calcium phosphate method essentially as described previously (19). Briefly, 293T cells were transfected with $10 \mu \mathrm{g}$ of the full-length proviral SIVmac239 harboring the luciferase gene in place of nef or cotransfected with $10 \mathrm{ng}$ of an env-defective reporter virus $(41,66 \mathrm{a})$ and $5 \mu \mathrm{g}$ of envelope expression vectors. After overnight incubation the medium was changed and virus was harvested $24 \mathrm{~h}$ later. Viral stocks were aliquoted and frozen at $-80^{\circ} \mathrm{C}$, and p27 antigen concentrations of viral stocks were quantitated with an SIV p27 antigen capture enzyme-linked immunosorbent assay kit obtained through the National Institutes of Health AIDS Research and Reference Reagent Program.

Cell culture. 293T cells were grown in Dulbecco modified Eagle medium supplemented with $10 \%$ fetal calf serum (FCS) and antibiotics. 221 cells were maintained in RPMI 1640 medium containing 20\% FCS, $100 \mathrm{U}$ of interleukin$2 / \mathrm{ml}$, and antibiotics as described previously (1).
Entry assays. To determine coreceptor activity of the STRL33 mutants, 293T cells were transiently cotransfected with CD4 and coreceptor expression plasmids. After overnight incubation the medium was changed and the cells were seeded in 48-well dishes. The following day, cells were infected with $50 \mathrm{ng}$ of luciferase reporter virus $(41,60)$ in a total volume of $0.5 \mathrm{ml}$. At 3 days after infection cells were lysed, and the luciferase activities in $20-\mu l$ cell lysate samples were determined using a commercially available kit (Promega). For infection with green fluorescent protein (GFP) reporter viruses (Sharron et al., submitted), $293 \mathrm{~T}$ cells were seeded in 12-well dishes. After overnight incubation, cells were cotransfected with CD4 and coreceptor expression plasmids. The following day, cells were infected with GFP reporter virus containing $100 \mathrm{ng}$ of p27 antigen in a total volume of $1 \mathrm{ml}$. Three days after infection cells were detached from the plates, washed and fixed with $2 \%$ paraformaldehyde and the percentage of GFP-positive cells was analyzed by fluorescence-activated cell sorting (FACS).

Western blot analysis. 293T cells were cotransfected with $10 \mu \mathrm{g}$ of STRL33 vectors containing a C-terminal AU1 tag. After overnight incubation the medium was replaced by fresh Dulbecco modified Eagle medium. Cells were harvested $48 \mathrm{~h}$ after transfection and lysed with lysis buffer $(0.5 \%$ Nonidet P-40, $0.15 \mathrm{M}$ $\mathrm{NaCl}, 50 \mathrm{mM}$ HEPES buffer [pH 7.5]) containing $10 \mathrm{mM} \mathrm{NaF}$ and $1 \mathrm{mM}$ phenylmethylsulfonyl fluoride (Sigma Chemicals, St. Louis, Mo.). Expression of STRL33 in cleared lysates was analyzed by immunoblotting. Proteins were detected with a 1:10,000 dilution of anti-AU1 antibody (Babco, Richmond, Calif.).

Epitope mapping. The STRL33-specific monoclonal antibody (MAb) 699 (mouse anti-STRL33 clone 56811; R\&D Systems, Minneapolis, Minn.) was generated as described previously $(43,66 \mathrm{a})$. For epitope mapping $293 \mathrm{~T}$ cells were seeded in 12-well dishes and transiently transfected with the various STRL33 expression vectors on the following day. At $18 \mathrm{~h}$ after transfection, cells were detached from the plate with $5 \mathrm{mM}$ EDTA and stained with phycoerythrinconjugated anti-STRL33 MAb 699 (66a). FACS analysis was performed essentially as described previously $(44,66 \mathrm{a})$.

Nucleotide sequence accession number. The smSTRL33 nucleotide sequence has been assigned GenBank accession no. AF237559.

\section{RESULTS}

SIVmac efficiently infects cells expressing huSTRL33 but not rhSTRL33. SIVmac239 is a well-characterized pathogenic molecular clone $(40,62)$ which has been used in many studies on the pathogenesis of primate lentiviruses and on vaccine development. It has been previously shown that SIVmac239 enters efficiently into macrophages but produces only very small amounts of progeny virus $(52,53)$. SIVmac239 uses both macaque and human CCR5 $(12,41,48)$ and human-derived GPR15 and STRL33 $(20,28,31)$. To investigate if the coreceptor tropism of SIVmac239 is species dependent, 293T cells were cotransfected with expression plasmids for CD4 and entry cofactor CCR5, GPR15, or STRL33 of both human and rhesus macaque origin. Subsequently, the cells were infected with replication-competent SIVmac239 carrying the luciferase reporter gene in place of the nef gene. A chimeric SIV with the env gene of an amphotropic murine leukemia virus named MuSIV-Luc (63) served as a positive control. MuSIV-Luc efficiently infected cells independently of CD4 or coreceptor expression (Fig. 1 and data not shown). In contrast, no luciferase activities were detected after infection with $e n v$-defective SIVmac239. SIVmac239 entered efficiently into cells expressing both human- and rhesus macaque-derived CCR5 and GPR15 (Fig. 1). Entry efficiencies observed with huSTRL33 were comparable to those observed with CCR5 and GPR15. Unexpectedly, however, about 13-fold-reduced entry levels were measured with cells expressing rhSTRL33 (Fig. 1).

The failure of rhSTRL33 to support efficient SIV infection could be due to structural differences between the rhesus macaque and human receptors or to inefficient expression of rhSTRL33. Therefore, we investigated if expression levels contributed to the different entry efficiencies. Since our antihuSTRL33-specific MAb does not cross-react with rhSTRL33 (66a), an AU1 tag was added to the C termini of the human and rhesus macaque proteins. We found that the AU1 tag did not impair the ability of huSTRL33 to function as an entry cofactor for SIVmac239 (Fig. 2A). However, similar to the results obtained with untagged rhSTRL33, about 25-fold- 


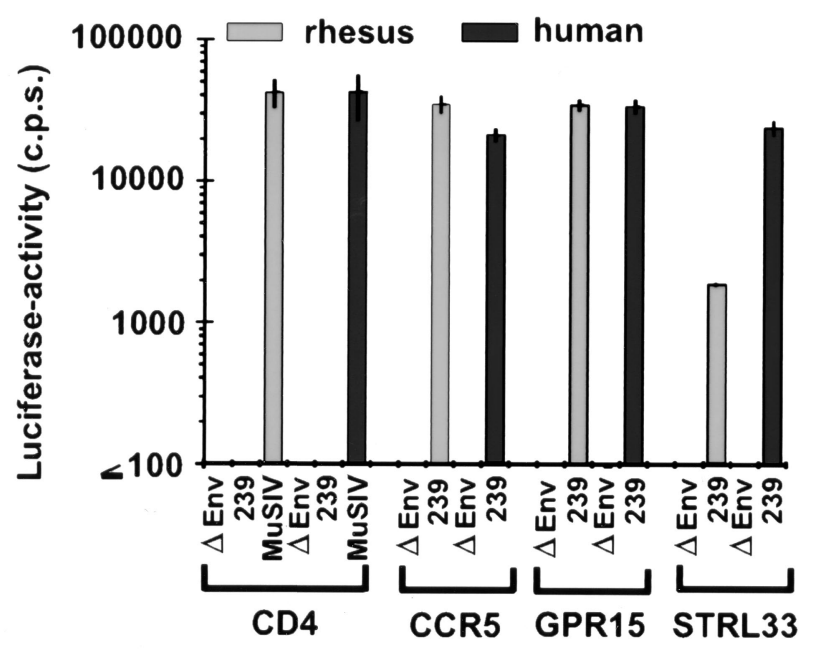

FIG. 1. SIVmac239 does not use rhSTRL33 for efficient entry. 293T cells were cotransfected with plasmids expressing human or macaque CD4 and the indicated entry cofactors of human and rhesus macaque origin. At 1 day posttransfection the cells were detached from the plates, seeded in 48 -well dishes, and infected in triplicate with intact luciferase reporter viruses containing $50 \mathrm{ng}$ of p27 antigen. Luciferase activities in the cellular extracts were measured at 3 days postinfection. Error bars give standard deviations from average values measured in three independent infections. Similar results were obtained in three additional experiments. As controls the transfected cells were infected with envelope-deleted SIVmac239 ( $\Delta$ env) or with a chimeric SIV containing the murine leukemia virus glycoprotein (MuSIV) (63). Luciferase values obtained with rhSTRL33 $(1,885 \pm 61)$ were about 13 -fold lower than those obtained with huSTRL33 $(24,043 \pm 2,993)$.

lower entry efficiencies were observed with AU1-tagged rhSTRL33. Western blot analysis revealed that huSTRL33 and rhSTRL33 proteins were expressed with comparable efficiencies (Fig. 2B). Therefore, species-specific differences rather than expression levels accounted for the failure of rhSTRL33 to support efficient SIV infection.

Variations in the $\mathbf{N}$ terminus of STRL33 determine SIVmac coreceptor function. It was unexpected that SIVmac239 was impaired in the utilization of STRL33 derived from its host, the rhesus macaque, but was capable of using huSTRL33 for efficient entry. Sequence alignments revealed that huSTRL33 and rhSTRL33 show 94\% amino acid identity. As shown in Fig. 3 the extracellular sequences of both orphan receptors differ at seven amino acid positions close to the $\mathrm{N}$ terminus and in a single residue in each of the three extracellular loops. As a first step to elucidate which specific amino acid variations were responsible for the species specificity of STRL33 coreceptor usage, we generated two AU1-tagged recombinants between huSTRL33 and rhSTRL33 (Fig. 4A). Exchanging the first 64 amino acids of huSTRL33 for the corresponding rhesus macaque-derived sequences impaired coreceptor function. The reciprocal construct, in which the N-terminal 64 amino acids of huSTRL33 were introduced into rhSTRL33, conferred full activity to rhSTRL33 (Fig. 4B). Western blot analysis revealed that both chimeric proteins were expressed equally well (data not shown). Thus, sequence variations close to the $\mathrm{N}$ terminus of STRL33 determined coreceptor activity.

Amino acid variation S31R determines STRL33 function as an SIV entry cofactor. The N-terminal region of STRL33 which determines coreceptor function contains seven amino acid variations between the human and rhesus macaque orphan receptors (Fig. 3). Among these are substitutions in several tyrosine residues (H4Y, Y6H, H7Y, and Y10D). It has been previously shown that $\mathrm{N}$-terminal tyrosines are important for CCR5 coreceptor function $(32,33)$.
To map the specific amino acid residues that determined SIV coreceptor activity, a variety of AU1-tagged mutants of both rhSTRL33 and huSTRL33 were analyzed (Fig. 5). Changes of Y4H, H6Y, Y7H, D10Y, L13•, and S14N in rhSTRL33 did not significantly increase its functional activity as an SIV coreceptor (Fig. 5, upper panel). In contrast, the R31S substitution resulted in entry efficiencies comparable to those observed with huSTRL33. The reverse substitutions in huSTRL33 confirmed the importance of the S31R substitution for coreceptor function: changes of $\mathrm{H} 4 \mathrm{Y}, \mathrm{Y} 6 \mathrm{H}, \mathrm{H} 7 \mathrm{Y}$, and Y10D had only moderate effects, whereas the S30R mutation resulted in about 20 -fold-reduced activity, comparable to that with rhSTRL33 (Fig. 5, lower panel). Western blot analysis revealed that, with the exception of the inactive $\Delta$ Nter huSTRL33 variant, all mutated coreceptors were expressed with comparable efficiencies (data not shown). Thus, the R31S substitution located just outside of the first transmembrane domain (Fig. 3) determined the differential coreceptor activities of huSTRL33 and rhSTRL33.

Several additional SIV envelopes were tested to exclude the possibility that the inefficient usage of rhSTRL33 as a coreceptor is specific for the SIVmac239 molecular clone. SIVmac316 is a derivative of SIVmac239 that replicates with high efficiency in alveolar macrophages $(52,53)$. It has been shown that macrophage- and T-cell-tropic SIV isolates interact differently with CCR5 (26). Consistent with the results obtained with SIVmac239, luciferase reporter viruses pseudotyped with the SIVmac316 envelope entered cells expressing huSTRL33 with higher efficiency than that with which they entered cells expressing rhSTRL33 (data not shown). Similar results were obtained with GFP reporter viruses pseudotyped with envelopes from SIVmacBK28, which was derived from SIVmac251 after propagation on the human T-cell line H9 (56); SIVsm $\Delta B 670$ cl.3, from transplacentally infected macaque infants (4); and the neurovirulent SIVmac17E/Fr strain $(5,27)$. The absolute number of GFP-positive cells coexpressing CD4 and huSTRL33 varied from $11.6 \%$ (SIVmacBK28) to $2.9 \%$ (SIVsm $\Delta \mathrm{B} 670 \mathrm{cl} .3$ )

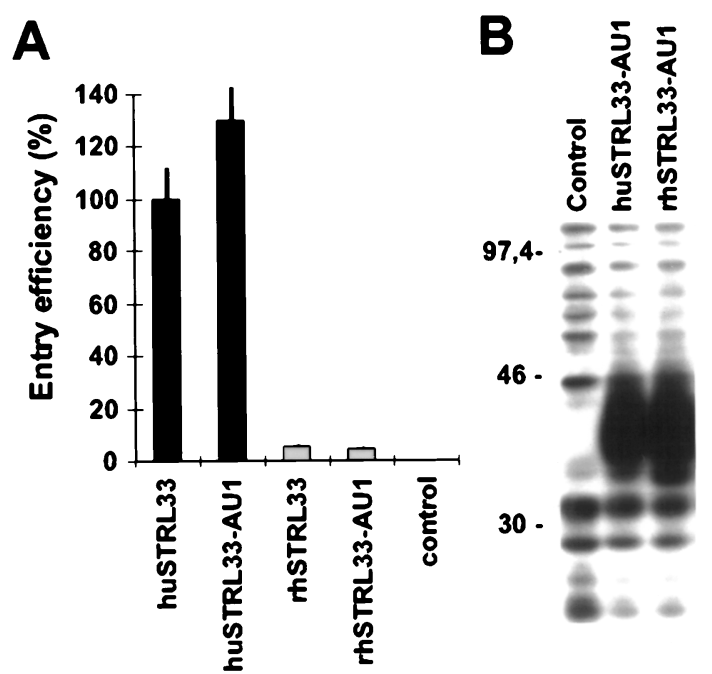

FIG. 2. Different SIV entry efficiencies are not due to inefficient expression of rhSTRL33. (A) 293T cells were cotransfected with CD4 and expression constructs for wild-type and AU1-tagged huSTRL33 and rhSTRL33. SIVmac239 entry was determined as described in the legend to Fig. 1. The results represent average values of three independent infections. (B) 293T cells were transfected with vectors expressing AU1-tagged huSTRL33 or rhSTRL33. Protein expression was verified by Western blot analysis as described in Materials and Methods. Control, cell extracts derived from mock-transfected 293T cells. 


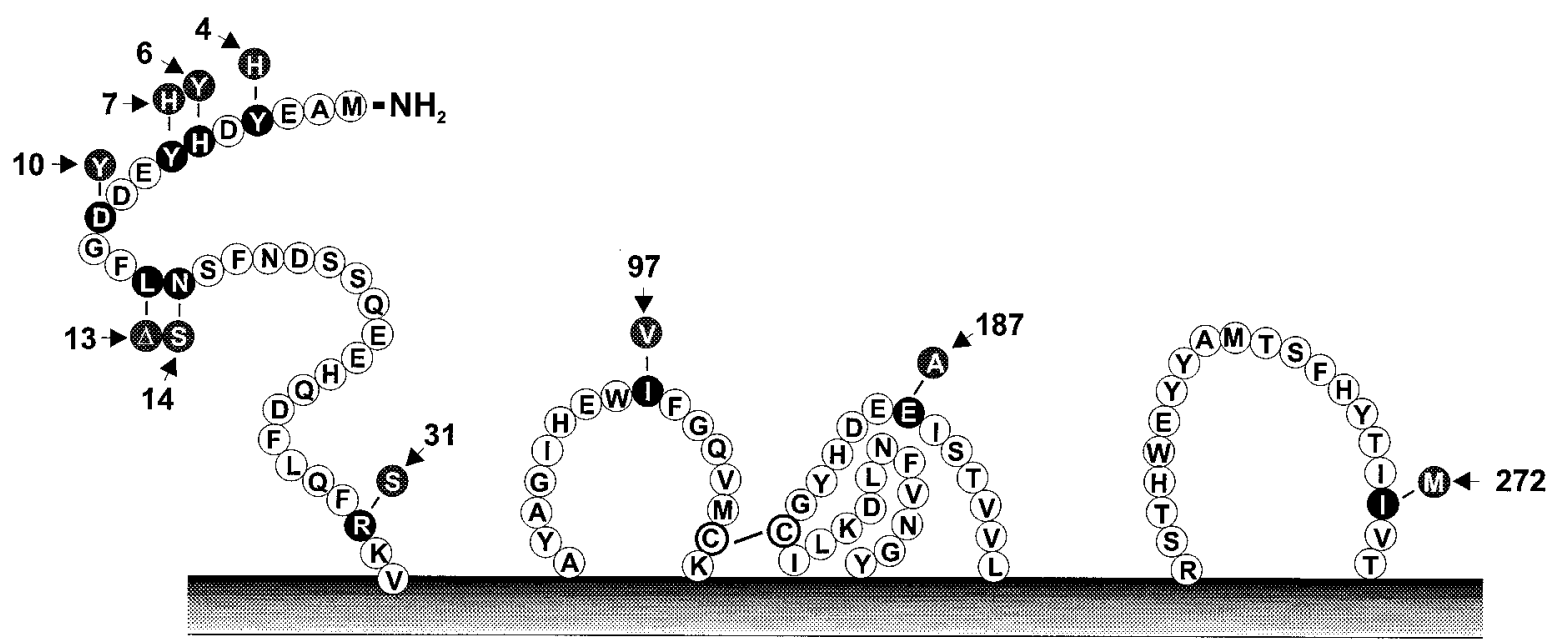

FIG. 3. Schematic presentation of the N-terminal region and the extracellular domains of rhSTRL33. Amino acid variations in huSTRL33 compared to the rhSTRL33 sequence are indicated. huSTRL33 and rhSTRL33 are 94\% identical at the amino acid level, with most differences clustered at the amino terminus (20, 46). Differences between huSTRL33 and rhSTRL33 are indicated by the shaded residues. The numbers give the amino acid positions in rhSTRL33. Bar, cell membrane.

to $0.8 \%$ (SIVmac17E/F). For all three envelopes the number of GFP-positive cells was 10- to 50-fold lower after transfection with rhSTRL33 (Fig. 6). Similar to the results obtained with SIVmac239 (Fig. 5), changes of H4Y, Y6H, H7Y, and Y10D close to the $\mathrm{N}$ terminus of huSTRL33 reduced coreceptor function only two- to fourfold (hu-mutNter; Fig. 6). In comparison, the S30R mutation in huSTRL33 had strongly disruptive effects and, conversely, the single R31S substitution in rhSTRL33 imparted coreceptor activity that was equivalent to that observed with huSTRL33 (Fig. 6). SIVsm $\Delta$ B670 cl.3 and SIVmac17E/F entered cells transfected with the huNter/ rhCter-STRL33 construct about two- to threefold more efficiently than cells transfected with the huSTRL33 expression vector. This indicates that variations in the three extracellular domains also contribute to STRL33 function as an SIV coreceptor. Nonetheless, the serine residue located just outside of the first transmembrane domain was clearly identified as the key residue for STRL33 coreceptor activity. We previously reported on the expression and coreceptor activity of STRL33 on human peripheral blood lymphocytes using a MAb against huSTRL33 (66a). We found that MAb 699 was unable to recognize rhSTRL33 and to block viral infection via huSTRL33 (66a). FACS analysis revealed that the epitope of MAb 699 maps to the N terminus of STRL33 but does not include the serine residue crucial for STRL33's coreceptor activity (data not shown). This likely accounts for its inability to block infection via huSTRL33. Since this MAb does not react in Western blot analysis, it probably recognizes a discontinuous or conformational epitope present in the $\mathrm{N}$ terminus of cell surfaceassociated huSTRL33.

SMSTRL33 is efficiently used as an SIV coreceptor. Both HIV-2 and SIVmac originate from sooty mangabeys naturally infected in west Africa $(35,36)$. To investigate whether STRL33 might play a role in SIV replication in mangabeys, we amplified the STRL33 open reading frame from three different animals by PCR. The predicted smSTRL33 amino acid sequence differed at a total of 19 positions $(\mathrm{H} 4 \mathrm{Y}, \mathrm{Y} 6 \mathrm{H}$, H7Y, Y10D, G11E, •13F, S14N, E22K, V97I, S105T, I109V, T152I, S153C, G182R, A187E, M246T, M251V, F253L, M272I) from the huSTRL33 sequence. Overall, the N-terminal region of smSTRL33 showed higher sequence similarity to rhSTRL33 than to huSTRL33. For example, the location of
N-terminal tyrosines was well conserved between STRL33 derived from both monkey species (Fig. 7A). Similar to huSTRL33, however, smSTRL33 contained a serine at amino acid position 31 (Fig. 7A). Functional analysis revealed that luciferase reporter viruses pseudotyped with the SIVmac239, SIVmac316, and SIVsm $\Delta$ B670 cl.3 envelopes entered cells expressing smSTRL33 or huSTRL33 with comparable efficiencies (Fig. 7B). The coreceptor activity of smSTRL33 indicates that it may be a relevant entry cofactor in naturally infected mangabeys and further supports the critical role of the serine residue for STRL33 coreceptor function.

\section{DISCUSSION}

In this study, we show that several SIVmac and SIVsm isolates use huSTRL33 and smSTRL33 as an entry cofactor with much higher efficiency than they use rhSTRL33. The func-

A

Ant

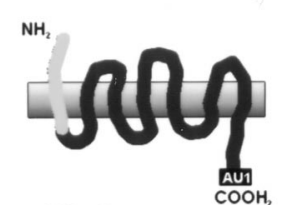

rhN/huC

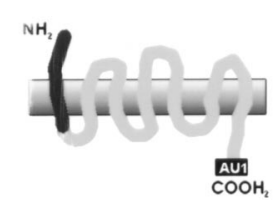

B

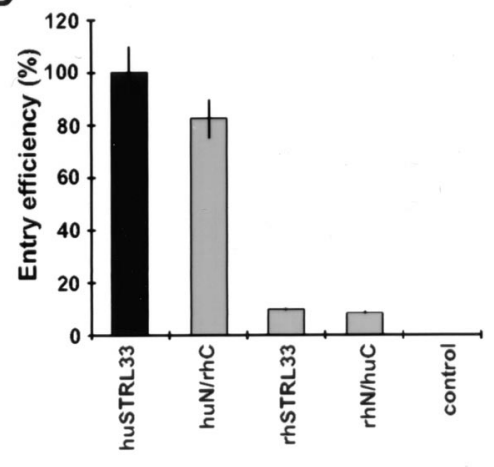

FIG. 4. Species-specific sequence variations in the $\mathrm{N}$ terminus of STRL33 determine SIV coreceptor function. (A) Schematic presentation of the AU1tagged STRL33 recombinants with an huSTRL33 N terminus and a rhSTRL33 $\mathrm{C}$ terminus (huN/rhC) and with an rhSTRL33 N terminus and an huSTRL33 C terminus (rhN/huC). (B) 293T cells were cotransfected with CD4 and expression constructs for the indicated STRL33 variants. SIVmac239 entry was tested as described in the legend to Fig. 1. The results represent average values of three independent infections. Control, cell extracts derived from mock-transfected 293 T cells. 


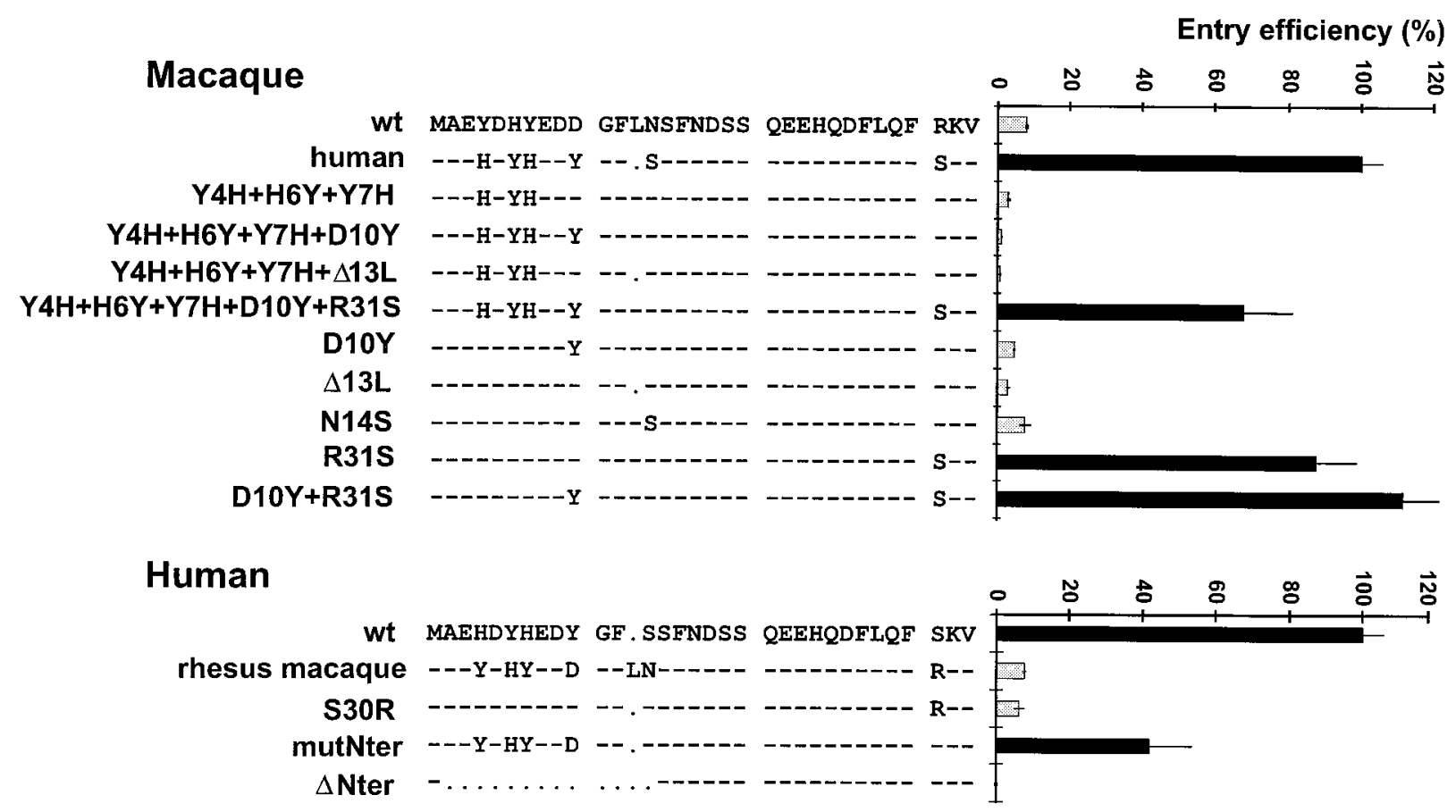

FIG. 5. Amino acid substitution of S31R impairs functional activity of rhSTRL33 as an SIVmac239 entry cofactor. Mutational analysis of rhSTRL33 and huSTRL33. The specific mutations compared to the original rhSTRL33 and huSTRL33 amino acid sequences are shown at the left. Dashes indicate amino acid identity, and dots indicate gaps. The relative entry efficiencies compared to human STRL33 are shown at the right. Average values from three infections are shown, and comparable results were obtained with independent virus stocks.

tional differences mapped to the $\mathrm{N}$ terminus of STRL33, which differs at seven positions between human and rhesus macaquederived coreceptors: H4Y, Y6H, H7Y, Y10D, •13L, N14S, and S31R (Fig. 3). Mutational analysis revealed that the S31R amino acid variation was of critical importance for coreceptor activity, irrespective of the SIV isolate tested. In comparison, sequence variations in N-terminal tyrosine residues between rhSTRL33 and huSTRL33 had only moderate effects.

huSTRL33 is efficiently used by many SIV isolates derived from infected rhesus macaques, and it has been suggested that this entry cofactor might play a role in the pathogenesis of primate lentiviruses $(20,31)$. Therefore, it was striking that rhSTRL33 was used very inefficiently compared to huSTRL33. Analysis of AU1-tagged orphan receptors revealed that huSTRL33 and rhSTRL33 were expressed with comparable efficiencies. Thus, the specific amino acid variations close to the $\mathrm{N}$ terminus of STRL33, and not different expression levels, determined coreceptor function. The finding that differences in $\mathrm{N}$-terminal sequences had a dramatic effect on the coreceptor function of STRL33 is in agreement with previous studies on CCR5 coreceptor function $(6,25,32,61,64)$. The N-terminal regions of SIV and HIV entry cofactors contain several tyrosines and acidic amino acid residues which are involved in coreceptor activity, and it has been suggested that tyrosine sulfation of the $\mathrm{N}$ terminus of CCR5 is important for HIV-1 entry $(32,33)$. Similarly to CCR5, both huSTRL33 and rhSTRL33 contain several acidic residues and two tyrosines close to the $\mathrm{N}$ terminus. The tyrosines are located at positions 6 and 10 in huSTRL33 and at positions 4 and 7 in rhSTRL33 (Fig. 3). These variations in the acidic and tyrosine-rich region, however, had only moderate effects on coreceptor activity. In comparison, an S30R substitution in huSTRL33 impaired coreceptor activity and the reverse R31S change in rhSTRL33 greatly increased coreceptor activity. Serine residues are also present at the $\mathrm{N}$ termini of several other coreceptors, and it has been shown that serine 17 in CCR5 is also important for viral entry (61). Therefore, serine residues may contribute to the coreceptor function of several 7TM GPCRs.

Overall, primate coreceptors are highly conserved, and usually the sequences derived from nonhuman primates and the corresponding human genes show $\geq 94 \%$ homology $(7,10,50)$. However, most of the differences are usually found in the $\mathrm{N}$ termini of the 7TM GPCRs. Some N-terminal sequence variations which could potentially influence coreceptor function are also found in the major HIV and SIV entry cofactors, CCR5 and CXCR4, derived from different primate species (7, 10, 72). The finding that rhSTRL33 was used much less efficiently than huSTRL33 underlines the importance of using coreceptors derived from the appropriate species when studying coreceptor tropism of primate lentiviruses. STRL33 derived from humans, sooty mangabeys, and African green monkeys (Cercopithecus aethiopis) contain a serine residue at position 31, whereas both Macaca mulatta and Macaca nemestrina contain an arginine at the corresponding position (Fig. 7A). African green monkey SIV (SIVagm) can use huSTRL33 (50), and it is possible that STRL33 is a relevant entry cofactor of the SIVagm group of primate lentiviruses. SIVmac and SIVsm do not utilize rhSTRL33 for efficient entry, and the S31R sequence variation that impaired coreceptor function is found in both $M$. mulatta- and M. nemestrina-derived STRL33. These results suggest that STRL33 is not of major importance for SIV replication and pathogenicity in experimentally infected macaques. These findings clearly do not rule out the possibility that STRL33 plays an important role in certain aspects of SIV and HIV pathogenicity and transmission. Our results also show that smSTRL33 is an efficient coreceptor for SIV, indicating that this orphan receptor might contribute to 


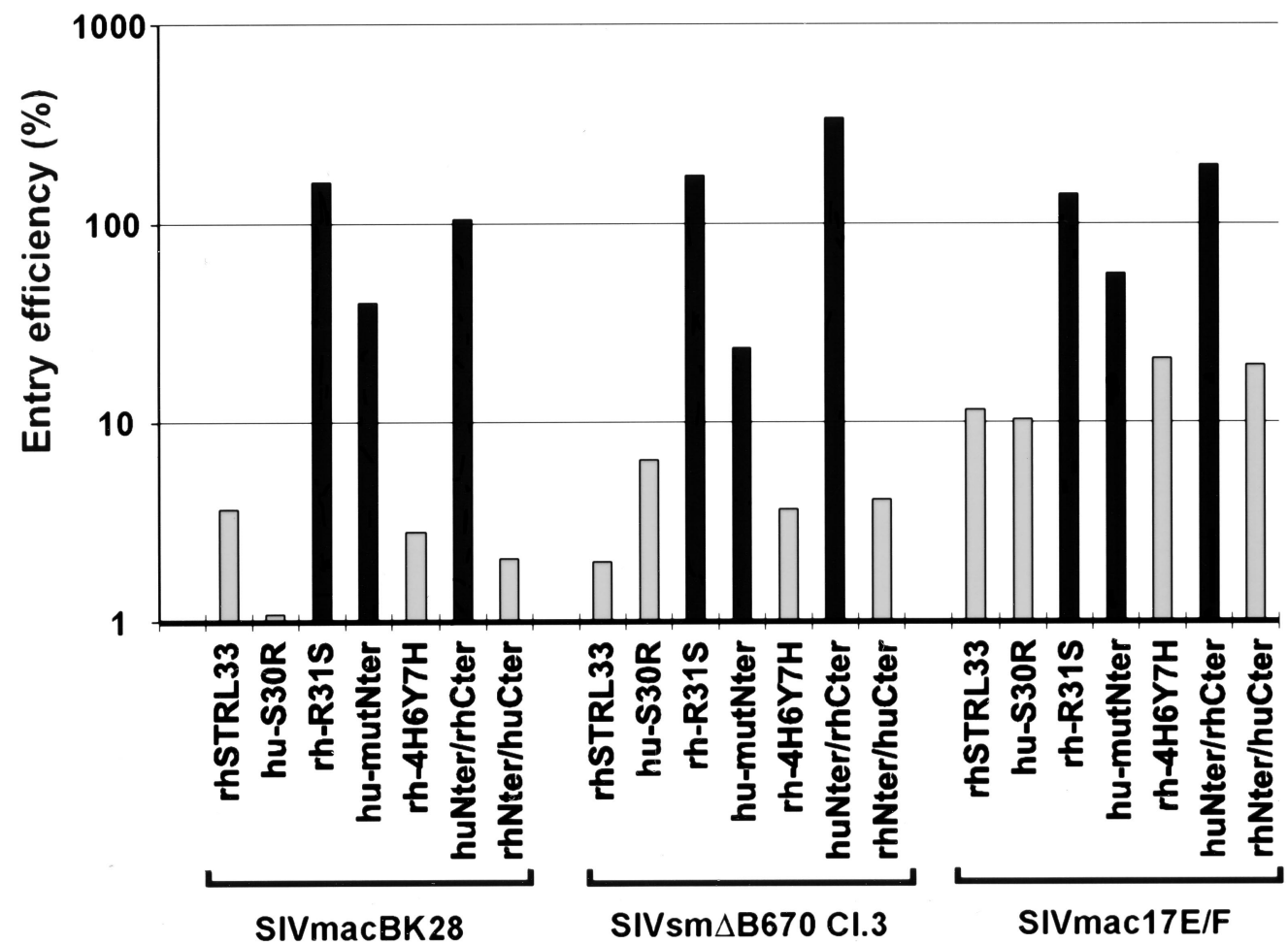

FIG. 6. Serine 31 is critical for STRL33 coreceptor function of several SIV isolates. SIVmacBK28, SIVsm $\Delta$ B670 cl.3, and SIVmac17E/F Env-pseudotyped GFP reporter viruses were used to infect cells coexpressing CD4 and the indicated STRL33 variants. Infection efficiency (percentage of GFP-positive cells) is shown relative to that obtained for huSTRL33 $(100 \%)$. Comparable results were obtained in two independent experiments.

viral replication in naturally infected mangabeys. Recent results suggest that some HIV-1 isolates might utilize STRL33 as a coreceptor on primary cells (66a). HIV-1 isolates derived from a mother-infant pair efficiently used STRL33 (70, 71), suggesting that this orphan receptor might play a role in vertical HIV-1 transmission. Furthermore, STRL33 was used relatively frequently by lung lymphocyte-derived clones isolated from one HIV-1-infected patient (67). The exact role of

A
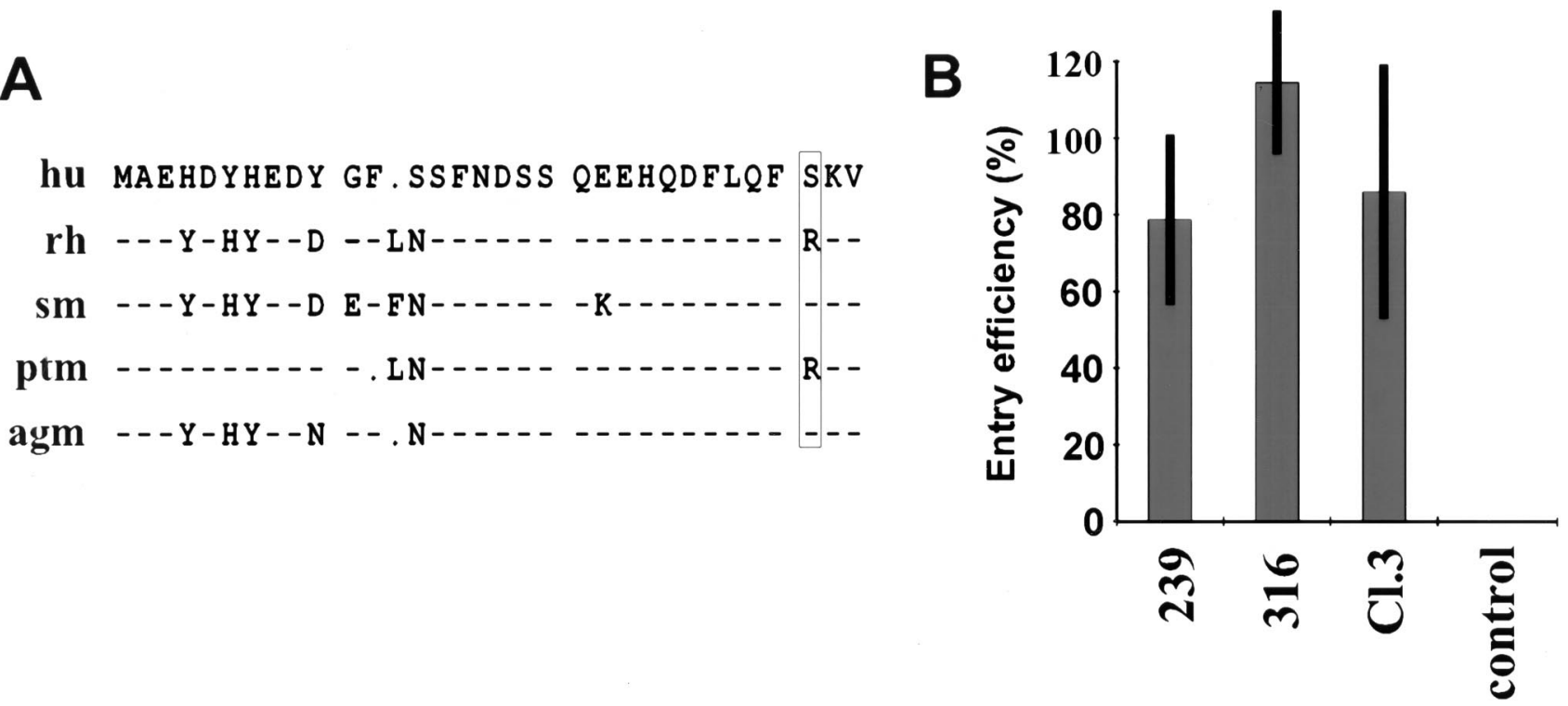

FIG 7. smSTRL 33 mediates efficient SIV entry. (A) Sequence variations at the $\mathrm{N}$ terminus of STRL33 derived from humans (hu), rhesus macaques (rh), sooty mangabeys (sm), pig tail macaques (ptm), and African green monkeys (agm) $(20,46)$. Dashes, identity with the human-derived STRL33 sequence; dots, gaps introduced to optimize the alignment. The position of the serine residue, which is critical for STRL33 coreceptor function, is boxed. (B) Entry of luciferase reporter viruses pseudotyped with SIVmac239, SIVmac316, and SIVsm $\Delta$ B670 cl.3 Env into cells coexpressing CD4 and smSTRL33. Infections were performed as indicated in Materials and Methods. Entry efficiency is shown relative to that obtained for huSTRL33. Data represent average values obtained for three independent infections. Control, cell extracts derived from mock-transfected $293 \mathrm{~T}$ cells infected with pseudotyped luciferase reporter viruses. 
STRL33 in the pathogenesis of primate immunodeficiency viruses, however, remains to be elucidated.

In summary, our findings suggest that STRL33 is not an important coreceptor in the SIV/macaque model but may play a role in naturally infected sooty mangabeys. Coreceptor expression levels are important for efficient viral entry (42, 44, 58). Recent findings suggest that STRL33 requires higher expression levels than CCR5 for mediating infection (44, 59, 66a). Importantly, rhSTRL33 was inefficient in mediating SIV entry even in transiently transfected $293 \mathrm{~T}$ cells that express very high STRL33 levels. Recently, it has been shown that GPR15 coreceptor usage in addition to CCR5 is of limited relevance for SIV replication in vivo (60). Taken together these results underline the central importance of CCR5 as a cofactor for SIV entry. Additional studies are required, however, to clarify if viral replication via these alternative receptors might induce immunodeficiency when CCR5 and CXCR4 are efficiently blocked.

\section{ACKNOWLEDGMENTS}

A number of reagents were obtained through the AIDS Research and Reference Reagents Program, Division of AIDS, NIAID, NIH. We thank Bernhard Fleckenstein for constant support and encouragement. We also thank Toshiaki Kodama for the full-length SIVmac239 clone.

This work was supported by the Deutsche Forschungsgemeinschaft (SFB 466) and the Johannes and Frieda Marohn Foundation. B.L. was supported by a K08 grant from the National Heart, Lung, and Blood Institute (HL03923-01) and the Measey Foundation Fellowship for Clinicians (Wistar Institute). R.W.D. is an Elizabeth Glazer Scientist of the Pediatric AIDS Foundation and was supported by National Institutes of Health grant R01 40880 and by a Burroughs Wellcome Fund award for translational research.

\section{REFERENCES}

1. Alexander, L., Z. Du, M. Rosenzweig, J. J. Jung, and R. C. Desrosiers. 1997. A role for natural simian immunodeficiency virus and human immunodeficiency virus type 1 nef alleles in lymphocyte activation. J. Virol. 71:60946099 .

2. Alkhatib, G., C. Combadiere, C. C. Broder, Y. Feng, P. E. Kennedy, P. M. Murphy, and E. A. Berger. 1996. CC-CKR5: a RANTES, MIP-1alpha, MIP1beta receptor as a fusion cofactor for macrophage-tropic HIV-1. Science 272:1955-1958.

3. Alkhatib, G., F. Liao, E. A. Berger, J. M. Farber, and K. W. Peden. 1997. A new SIV co-receptor, STRL33. Nature 388:238.

4. Amedee, A. M., N. Lacour, J. L. Gierman, L. N. Martin, J. E. Clements, R. Bohm, Jr., R. M. Harrison, and M. Murphey-Corb. 1995. Genotypic selection of simian immunodeficiency virus in macaque infants infected transplacentally. J. Virol. 69:7982-7990.

5. Anderson, M. G., D. Hauer, D. P. Sharma, S. V. Joag, O. Narayan, M. C. Zink, and J. E. Clements. 1993. Analysis of envelope changes acquired by SIVmac239 during neuroadaption in rhesus macaques. Virology 195:616626.

6. Atchison, R. E., J. Gosling, F. S. Monteclaro, C. Franci, L. Digilio, I. F. Charo, and M. A. Goldsmith. 1996. Multiple extracellular elements of CCR-5 and HIV-1 entry: dissociation from response to chemokines. Science 274:1924-1926.

7. Benton, P. A., D. R. Lee, and R. C. Kennedy. 1998. Sequence comparisons of non-human primate HIV-1 coreceptor homologues. Mol. Immunol. 35:95101.

8. Berger, E. A., P. M. Murphy, and J. M. Farber. 1999. Chemokine receptors as HIV-1 coreceptors: roles in viral entry, tropism, and disease. Annu. Rev. Immunol. 17:657-700.

9. Bieniasz, P. D., and B. R. Cullen. 1998. Chemokine receptors and human immunodeficiency virus infection. Front. Biosci. 3:44-58.

10. Carrington, M., T. Kissner, B. Gerrard, S. Ivanov, S. J. O'Brien, and M. Dean. 1997. Novel alleles of the chemokine-receptor gene CCR5. Am. J. Hum. Genet. 61:1261-1267.

11. Chakrabarti, L., M. Guyader, M. Alizon, M. D. Daniel, R. C. Desrosiers, P. Tiollais, and P. Sonigo. 1987. Sequence of simian immunodeficiency virus from macaque and its relationship to other human and simian retroviruses. Nature 328:543-547.

12. Chen, Z., P. Zhou, D. D. Ho, N. R. Landau, and P. A. Marx. 1997. Genetically divergent strains of simian immunodeficiency virus use CCR5 as a corecep- tor for entry. J. Virol. 71:2705-2714.

13. Chen, Z., A. Gettie, D. D. Ho, and P. A. Marx. 1998. Primary SIVsm isolates use the CCR5 coreceptor from sooty mangabeys naturally infected in west Africa: a comparison of coreceptor usage of primary SIVsm, HIV-2, and SIVmac. Virology 246:113-124.

14. Chen, Z., D. Kwon, Z. Jin, S. Monard, P. Telfer, M. S. Jones, C. Y. Lu, R. F. Aguilar, D. D. Ho, and P. A. Marx. 1998. Natural infection of a homozygous delta 24 CCR5 red-capped mangabey with an R2b-tropic simian immunodeficiency virus. J. Exp. Med. 188:2057-2065.

15. Choe, H., M. Farzan, Y. Sun, N. Sullivan, B. Rollins, P. D. Ponath, L. Wu, C. R. Mackay, G. LaRosa, W. Newman, N. Gerard, C. Gerard, and J. Sodroski. 1996. The beta-chemokine receptors CCR3 and CCR5 facilitate infection by primary HIV-1 isolates. Cell $\mathbf{8 5}: 1135-1148$.

16. Choe, H., M. Farzan, M. Konkel, K. Martin, Y. Sun, L. Marcon, M. Cayabyab, M. Berman, M. E. Dorf, N. Gerard, C. Gerard, and J. Sodroski. 1998. The orphan seven-transmembrane receptor Apj supports the entry of primary T-cell-line-tropic and dualtropic human immunodeficiency virus type 1 . J. Virol. 72:6113-6118.

17. Daniel, M. D., N. L. Letvin, N. W. King, M. Kannagi, P. K. Sehgal, R. D. Hunt, P. J. Kanki, M. Essex, and R. C. Desrosiers. 1985. Isolation of T-cell tropic HTLV-III-like retrovirus from macaques. Science 228:1201-1204.

18. De Cock, K. M., G. Adjorlolo, E. Ekpini, T. Sibailly, J. Kouadio, M. Maran, K. Brattegaard, K. M. Vetter, R. Doorly, and H. D. Gayle. 1993. Epidemiology and transmission of HIV-2: why there is no HIV-2 pandemic. JAMA 270:2083-2086.

19. Deng, H., R. Liu, W. Ellmeier, S. Choe, D. Unutmaz, M. Burkhart, P. Di Marzio, S. Marmon, R. E. Sutton, C. M. Hill, C. B. Davis, S. C. Peiper, T. J. Schall, D. R. Littman, and N. R. Landau. 1996. Identification of a major co-receptor for primary isolates of HIV-1. Nature 381:661-666.

20. Deng, H. K., D. Unutmaz, V. N. Kewalramani, and D. R. Littman. 1997. Expression cloning of new receptors used by simian and human immunodeficiency viruses. Nature 388:296-300.

21. Desrosiers, R. C., and D. J. Ringler. 1989. Use of SIV for AIDS research. Intervirology 30:301-312.

22. Doms, R. W., and S. C. Peiper. 1997. Unwelcome guests with master keys: how HIV uses chemokine receptors for cellular entry. Virology 235:179-190.

23. Doranz, B. J., J. Rucker, Y. Yi, R. J. Smyth, M. Samson, S. C. Peiper, M. Parmentier, R. G. Collman, and R. W. Doms. 1996. A dual-tropic primary HIV-1 isolate that uses fusin and the beta-chemokine receptors CKR-5, CKR-3, and CKR-2b as fusion cofactors. Cell 85:1149-1158.

24. Dragic, T., V. Litwin, G. P. Allaway, S. R. Martin, Y. Huang, K. A. Nagashima, C. Cayanan, P. J. Maddon, R. A. Koup, J. P. Moore, W. A. Paxton. 1996. HIV-1 entry into CD4+ cells is mediated by the chemokine receptor CC-CKR-5. Nature 381:667-673.

25. Dragic, T., A. Trkola, S. W. Lin, P. A. Nagashima, F. Kajumo, L. Zhao, W. C. Olson, L. Wu, C. R. Mackay, G. P. Allaway, T. P. Sakmar, J. P. Moore, and P. J. Maddon. 1998. Amino-terminal substitutions in the CCR5 coreceptor impair gp120 binding and human immunodeficiency virus type 1 entry. J. Virol. 72:279-285.

26. Edinger, A. L., A. Amedee, K. Miller, B. J. Doranz, M. Endres, M. Sharron, M. Samson, Z. H. Lu, J. E. Clements, M. Murphey-Corb, S. C. Peiper, M. Parmentier, C. C. Broder, and R. W. Doms. 1997. Differential utilization of CCR5 by macrophage and $\mathrm{T}$ cell tropic simian immunodeficiency virus strains. Proc. Natl. Acad. Sci. USA 94:4005-4010.

27. Edinger, A. L., J. L. Mankowski, B. J. Doranz, B. J. Margulies, B. Lee, J. Rucker, M. Sharron, T. L. Hoffman, J. F. Berson, M. C. Zink, V. M. Hirsch, J. E. Clements, and R. W. Doms. 1997. CD4-independent, CCR5-dependent infection of brain capillary endothelial cells by a neurovirulent simian immunodeficiency virus strain. Proc. Natl. Acad. Sci. USA 94:14742-14747.

28. Edinger, A. L., T. L. Hoffman, M. Sharron, B. Lee, B. O'Dowd, and R. W. Doms. 1998. Use of GPR1, GPR15, and STRL33 as coreceptors by diverse human immunodeficiency virus type 1 and simian immunodeficiency virus envelope proteins. Virology 249:367-378.

29. Edinger, A. L., T. L. Hoffman, Y. Yi, M. Sharron, R. G. Collman, B. Mitrovic, D. Faulds, J. Hesselgesser, R. Horuk, and R. W. Doms. 1998. An orphan seven transmembrane domain receptor expressed widely in the brain functions as a coreceptor for HIV-1 and SIV. J. Virol. 72:7934-7940.

30. Edinger, A. L., C. Blanpain, K. J. Kunstman, S. M. Wolinsky, M. Parmentier, and R. W. Doms. 1999. Functional dissection of CCR5 coreceptor function through the use of CD4-independent simian immunodeficiency virus strains. J. Virol. 73:4062-4073.

31. Farzan, M., H. Choe, K. Martin, L. Marcon, W. Hofmann, G. Karlsson, Y. Sun, P. Barrett, N. Marchand, N. Sullivan, N. Gerard, C. Gerard, and J. Sodroski. 1997. Two orphan seven-transmembrane segment receptors which are expressed in CD4-positive cells support simian immunodeficiency virus infection. J. Exp. Med. 186:405-411.

32. Farzan, M., H. Choe, L. Vaca, K. Martin, Y. Sun, E. Desjardins, N. Ruffing, L. Wu, R. Wyatt, N. Gerard, C. Gerard, and J. Sodroski. 1998. A tyrosinerich region in the $\mathrm{N}$ terminus of CCR5 is important for human immunodeficiency virus type 1 entry and mediates an association between gp120 and CCR5. J. Virol. 72:1160-1164.

33. Farzan, M., T. Mirzabekov, P. Kolchinsky, R. Wyatt, M. Cayabyab, N. P. 
Gerard, C. Gerard, J. Sodroski, and H. Choe. 1999. Tyrosine sulfation of the amino terminus of CCR5 facilitates HIV-1 entry. Cell 96:667-676.

34. Feng, Y., C. C. Broder, P. E. Kennedy, and E. A. Berger. 1996. HIV-1 entry cofactor: functional cDNA cloning of a seven-transmembrane, G proteincoupled receptor. Science 272:872-877.

35. Gao, F., L. Yue, and A. T. White. 1992. Human infection by genetically diverse SIVSM-related HIV-2 in west Africa. Nature 358:495-499.

36. Gardner, M. B. 1996. The history of simian AIDS. J. Med. Primatol. 25:148 157.

37. Hill, C. M., H. Deng, D. Unutmaz, V. N. Kewalramani, L. Bastiani, M. K. Gorny, S. Zolla-Pazner, and D. R. Littman. 1997. Envelope glycoproteins from human immunodeficiency virus types 1 and 2 and simian immunodeficiency virus can use human CCR5 as a coreceptor for viral entry and make direct CD4-dependent interactions with this chemokine receptor. J. Virol. 71:6296-6304.

38. Horuk, R., J. Hesselgesser, Y. Zhou, D. Faulds, D. Taub, M. Samson, M. Parmentier, J. Rucker, B. J. Doranz, and R. W. Doms. 1998. The CC chemokine I309 is a functional ligand for ChemR1/CCR8 and inhibits ChemR1/CCR8 dependent infection by diverse HIV-1 strains. J. Biol. Chem. 273:386-391.

39. Johnson, P. R., and V. M. Hirsch. 1992. SIV infection of macaques as a model for AIDS pathogenesis. Int. Rev. Immunol. 8:55-63.

40. Kestler, H. W., T. Kodama, D. Ringler, M. Marthas, N. Pedersen, A. Lackner, D. Regier, P. Sehgal, M. D. Daniel, and R. C. Desrosiers. 1990. Induction of AIDS in rhesus monkeys by molecularly cloned simian immunodeficiency virus. Science 248:1109-1112.

41. Kirchhoff, F., S. Pöhlmann, M. Hamacher, R. E. Means, T. Kraus, K. Überla, and P. Di Marzio. 1997. Simian immunodeficiency virus variants with differential T-cell and macrophage tropism use CCR5, and an unidentified cofactor expressed in CEMx174 cells for efficient entry. J. Virol. 71:65096516.

42. Kozak, S. L., E. J. Platt, N. Madani, F. E. Ferro, K. Peden, and D. Kabat. 1997. CD4, CXCR-4, and CCR5 dependencies for infections by primary patient and laboratory-adapted isolates of human immunodeficiency virus type 1. J. Virol. 71:873-882

43. Lee, B., M. Sharron, C. Blanpain, B. J. Doranz, J. Vakili, P. Setoh, E. Berg, G. Liu, H. R. Guy, S. R. Durell, M. Parmentier, C. N. Chang, K. Price, M. Tsang, and R. W. Doms. 1999. Epitope mapping of CCR5 reveals multiple conformational states and distinct but overlapping structures involved in chemokine and coreceptor function. J. Biol. Chem. 274:9617-9626.

44. Lee, B., M. Sharron, L. J. Montaner, D. Weissman, and R. W. Doms. 1999. Quantification of CD4, CCR5, and CXCR4 levels on lymphocyte subsets, dendritic cells, and differentially conditioned monocyte-derived macrophages. Proc. Natl. Acad. Sci. USA 96:5215-5220.

45. Letvin, N. L., M. D. Daniel, P. K. Sehgal, R. C. Desrosiers, R. D. Hunt, L. M. Waldron, J. J. MacKey, D. K. Schmidt, L. V. Chalifoux, and N. W. King. 1985. Induction of AIDS-like disease in macaque monkeys with T-cell tropic retrovirus STLV-III. Science 230:71-73.

46. Liao, F., G. Alkhatib, K. W. Peden, G. Sharma, E. A. Berger, and J. M. Farber. 1997. STRL33, A novel chemokine receptor-like protein, functions as a fusion cofactor for both macrophage-tropic and T cell line-tropic HIV-1. J. Exp. Med. 185:2015-2023.

47. Loetscher, M., A. Amara, E. Oberlin, N. Brass, D. Legler, P. Loetscher, M. D’Apuzzo, E. Meese, D. Rousset, J. L. Virelizier, M. Baggiolini, F. ArenzanaSeisdedos, and B. Moser. 1997. TYMSTR, a putative chemokine receptor selectively expressed in activated T cells, exhibits HIV-1 coreceptor function. Curr. Biol. 7:652-660.

48. Marcon, L., H. Choe, K. Martin, M. Farzan, P. D. Ponath, L. Wu, W. Newman, N. Gerard, C. Gerard, and J. Sodroski. 1997. Utilization of C-C chemokine receptor 5 by the envelope glycoproteins of a pathogenic simian immunodeficiency virus, SIVmac239. J. Virol. 71:2522-2537.

49. Marlink, R. G., D. Ricard, S. M. Boup, P. J. Kanki, J. L. Romet-Lemonne, I. N. Doye, K. Diop, M. A. Simpson, F. Greco, M. J. Chou, V. Degruttola, C. C. Hsieh, C. Boye, F. Barin, F. Denis, M. F. McLane, and M. Essex. 1988. Clinical, hematological, and immunologic cross-sectional evaluation of individuals exposed to human immunodeficiency virus type-2 (HIV-2). AIDS Res. Hum. Retroviruses 4:137-148.

50. Marx, P. A., and Z. Chen. 1998. The function of simian chemokine receptors in the replication of SIV. Semin. Immunol. 10:215-223.

51. Moore, J. P., A. Trkola, and T. Dragic. 1997. Co-receptors for HIV-1 entry. Curr. Opin. Immunol. 9:551-562.

52. Mori, K., D. J. Ringler, T. Kodama, and R. C. Desrosiers. 1992. Complex determinants of macrophage tropism in env of simian immunodeficiency virus. J. Virol. 66:2067-2075.

53. Mori, K., D. J. Ringler, and R. C. Desrosiers. 1993. Restricted replication of simian immunodeficiency virus strain 239 in macrophages is determined by env but is not due to restricted entry. J. Virol. 67:2807-2814.
54. Mörner, A., A. Björndal, J. Albert, V. N. Kewalramani, D. R. Littman, R. Inoue, R. Thorstensson, E. M. Fenyo, and E. Bjorling. 1999. Primary human immunodeficiency virus type 2 (HIV-2) isolates, like HIV-1 isolates, frequently use CCR5 but show promiscuity in coreceptor usage. J. Virol. 73: 2343-2349.

55. Murphey-Corb, M., L. N. Martin, S. R. Rangan, G. B. Baskin, B. J. Gormus, R. H. Wolf, W. A. Andes, M. West, and R. C. Montelaro. 1986. Isolation of an HTLV-III related retrovirus from macaques with simian AIDS and its possible origin in asymptomatic mangabeys. Nature 321:435-437.

56. Naidu, Y. M., H. W. Kestler, Y. Li, C. V. Butler, D. P. Silva, D. K. Schmidt, C. D. Troup, P. K. Sehgal, P. Sonigo, M. D. Daniel, and R. C. Desrosiers. 1988. Characterization of infectious molecular clones of simian immunodeficiency virus (SIVmac) and human immunodeficiency virus type 2: persistent infection of rhesus monkeys with molecularly cloned SIVmac. J. Virol. 62:4691-4696.

57. Owen, S. M., D. Ellenberger, M. Rayfield, S. Wiktor, P. Michel, M. H. Grieco, F. Gao, B. H. Hahn, and R. B. Lal. 1998. Genetically divergent strains of human immunodeficiency virus type 2 use multiple coreceptors for viral entry. J. Virol. 72:5425-5432.

58. Platt, E. J., K. Wehrly, S. E. Kuhnman, B. Chesbro, and D. Kabat. 1998. Effects of CCR5 and CD4 cell surface concentrations on infections by macrophage-tropic isolates of human immunodeficiency virus type 1. J. Virol. 72: 2855-2864.

59. Pöhlmann, S., M. Krumbiegel, and F. Kirchhoff. 1999. Coreceptor usage of BOB/GPR-15 and Bonzo/STRL-33 by primary HIV-1 isolates. J. Gen. Virol. 80:1241-1251.

60. Pöhlmann, S., N. Stolte, J. Münch, P. Ten Haaft, J. L. Heeney, C. StahlHennig, and F. Kirchhoff. 1999. Co-receptor usage of BOB/GPR15 in addition to CCR5 has no significant effect on replication of simian immunodeficiency virus in vivo. J. Infect. Dis. 180:1494-1502.

61. Rabut, G. E., J. A. Konner, F. Kajumo, J. P. Moore, and T. Dragic. 1998. Alanine substitutions of polar and nonpolar residues in the amino-terminal domain of CCR5 differently impair entry of macrophage- and dualtropic isolates of human immunodeficiency virus type 1. J. Virol. 72:3464-3468.

62. Regier, D. A., and R. C. Desrosiers. 1990. The complete nucleotide sequence of a pathogenic molecular clone of SIV. AIDS Res. Hum. Retroviruses 6: 1221-1231.

63. Reiprich, S., B. R. Gundlach, B. Fleckenstein, and K. Überla. 1997. Replication-competent chimeric lenti-oncovirus with expanded host cell tropism. J. Virol. 71:3328-3331.

64. Ross, T. M., P. D. Bieniasz, and B. R. Cullen. 1998. Multiple residues contribute to the inability of murine CCR-5 to function as a coreceptor for macrophage-tropic human immunodeficiency virus type 1 isolates. J. Virol. 72:1918-1924.

65. Rucker, J., A. L. Edinger, M. Sharron, M. Samson, B. Lee, J. F. Berson, Y. Yi, B. Margulies, R. G. Collman, B. J. Doranz, M. Parmentier, and R. W. Doms. 1997. Utilization of chemokine receptors, orphan receptors, and herpesvirus-encoded receptors by diverse human and simian immunodeficiency viruses. J. Virol. 71:8999-9007.

66. Samson, M., A. L. Edinger, P. Stordeur, J. Rucker, V. Verhasselt, M. Sharron, C. Govaerts, C. Mollereau, G. Vassart, R. W. Doms, and M. Parmentier. 1998. ChemR23, a putative chemoattractant receptor, is expressed in dendritic cells and is a coreceptor for SIV and some primary HIV-1 strains. Eur. J. Immunol. 26:3021-3028.

66a.Sharron, M., S. Pöhlmann, M. Tsang, F. Kirchhoff, R. W. Doms, and B. Lee. Characterization of STRL33 expression and coreceptor activity. Blood, in press.

67. Singh, A., G. Besson, A. Mobasher, and R. G. Collman. 1999. Patterns of chemokine receptor fusion cofactor utilization by human immunodeficiency virus type 1 variants from the lungs and blood. J. Virol. 73:6680-6690.

68. Unutmaz, D., V. N. Kewalramani, and D. R. Littman. 1998. G proteincoupled receptors in HIV and SIV entry: new perspectives on lentivirus-host interactions and on the utility of animal models. Semin. Immunol. 10:225236.

69. Zhang, L, T. He, Y. Huang, Z Chen, Y. Guo, S. Wu, K. J. Kunstman, R. C. Brown, J. P. Phair, A. U. Neumann, D. D. Ho, and S. M. Wolinsky. 1998. Chemokine coreceptor usage by diverse primary isolates of human immunodeficiency virus type 1. J. Virol. 72:9307-9312.

70. Zhang, Y. J., T. Dragic, Y. Cao, L. Kostrikis, D. S. Kwon, D. R. Littman, V. N. Kewalramani, and J. P. Moore. 1998. Use of coreceptors other than CCR 5 by non-syncytium-inducing adult and pediatric isolates of human immunodeficiency virus type 1 is rare in vitro. J. Virol. 72:9337-9344.

71. Zhang, Y. J., and J. P. Moore. 1999. Will multiple coreceptors need to be targeted by inhibitors of human immunodeficiency virus type 1 entry? J. Virol. 73:3443-3448.

72. Zhang, Y. W., O. A. Ryder, and Y. P. Zhang. 1999. Sequence evolution of the CCR5 chemokine receptor gene in primates. Mol. Biol. Evol. 16:1145-1154. 